\title{
ON THE CALCULATION OF LATTICE SUMS
}

\author{
by B. R. A. NIJBOER and F. W. DE WETTE \\ Instituut voor theoretische Fysica, Rijksuniversiteit te Utrecht, Nederland
}

\section{Synopsis}

A short and straightforward method for the conversion of slowly converging lattice sums into expressions with good convergence is presented. As an illustration a wellknown expression for the Madelung constant is rederived. The method is then applied to two general types of lattice sums, the latter of which has not been treated before.

$\S 1$. Introduction. Lattice sums, i.e. summations over the sites of an infinite perfect lattice of some potential energy function or force interaction, occur in many branches of crystal physics. The appearance of lattice sums in calculations of the lattice energy of ionic crystals and in considerations on the stability of the various lattice types are the best known and also oldest examples. Other cases where the evaluation of these sums is of some importance are investigations of the electromagnetic, optical, or elastic properties of crystals.

A difficulty met with in the evaluation of lattice sums always has been the question of their convergence. It presents itself under two aspects, firstly the convergence of the series as such and secondly the rapidity of convergence, the latter question being mainly of practical importance. It is a well-known fact that interactions decreasing slowly with distance (e.g. the Coulomb interaction) can give rise to lattice sums which exhibit only a conditional convergence. Since in this case the sum of the series is not uniquely determined, physical arguments will have to be invoked in order to arrive at physically meaningful answers. But even in the case of unconditional convergence, the actual rapidity of convergence is generally so poor that the series as they stand, are not very useful for computations.

In the existing literature on lattice sums a great deal of attention has been devoted to these problems. The classic examples in this connection are the treatments of Madelung 1) *) (for the Madelung-constant) and of Ewald ${ }^{3}$ ) (for more general types of lattice sums), in which the application of a summation recipe, based on physical arguments, and the transformation of the lattice sum into a more rapidly converging form are closely connected. Still

*) An equivalent method has been developed by Ornstein and Zernike 2). 
another method has been proposed by Evjen $\left.{ }^{4}\right)$. Most of the later developments are generalizations or modifications of these methods. The present paper is not different in this respect. It gives a method for the evaluation of lattice sums which seems to be more straightforward and less complicated than the usual procedures. Although in some respects this method is quite similar to that of Misra ${ }^{5}$ ), it was in its present simple form first used for a special case by Placzek, Nijboer and Van Hove ${ }^{6}$ ) in connection with the theory of neutron scattering in dense systems*). It can be readily extended to apply to a wide class of lattice sums. In this paper we will, after some preliminaries, first illustrate the method by rederiving a familiar expression for the Madelung-constant ( $(3)$. Then, after a short exposition of the principle involved in this summation method ( $(4)$, we will proceed to apply the scheme to two more general types of lattice sums $(\S \S 5,6)$, the latter of which as far as we know, has not been treated before.

§ 2. Preliminaries. We will consider lattice sums of perfect simple lattices (Bravais lattices); the method can easily be generalized for composite lattices. The usual notation for Bravais lattices will be used.

The unit cell of the lattice is specified by the basic vectors $\mathbf{a}_{1}, \mathbf{a}_{2}, \mathbf{a}_{3}$. The volume of the unit cell is

$$
v_{a}=\mathbf{a}_{1} \cdot\left(\mathbf{a}_{2} \wedge \mathbf{a}_{3}\right) .
$$

The space vector $\mathbf{r}$ will be expressed in terms of the basic vectors

$$
\mathbf{r}=r_{1} \mathbf{a}_{1}+r_{2} \mathbf{a}_{2}+r_{3} \mathbf{a}_{3} \text {. }
$$

The origin is chosen at the origin of one of the unit cells, the zero cell. The position vector of any lattice point (lattice vector) is

$$
\mathbf{r}_{\lambda}=\lambda_{1} \mathbf{a}_{1}+\lambda_{2} \mathbf{a}_{2}+\lambda_{3} \mathbf{a}_{3}
$$

where $\lambda$ denotes the set $\lambda_{1}, \lambda_{2}, \lambda_{3}$ (pos. and neg. integers).

The unit cell of the reciprocal lattice is mapped out by the reciprocal lattice vectors $\mathbf{b}_{1}, \mathbf{b}_{2}, \mathbf{b}_{3}$, defined by the relations

$$
\mathbf{a}_{i} \cdot \mathbf{b}_{\jmath}=\delta_{i \jmath} .
$$

A vector in reciprocal space will be expressed in terms of the b's

$$
\mathbf{h}=h_{1} \mathbf{b}_{1}+h_{2} \mathbf{b}_{2}+h_{\mathbf{3}} \mathbf{b}_{3} \text {. }
$$

Similarly the reciprocal lattice vector is written as

$$
\mathbf{h}_{\lambda}=\lambda_{1} \mathbf{b}_{1}+\lambda_{2} \mathbf{b}_{2}+\lambda_{3} \mathbf{b}_{3}
$$

again $\lambda_{1}, \lambda_{2}, \lambda_{3}$ are positive and negative integers.

*) An equivalent treatment for the Madelung constant was also given by Bertaut ?). 
The lattice sums that will be considered here are summations over the sites of an infinite perfect simple lattice of a potential energy function of the general form $\phi(\mathbf{r}-\mathbf{R}) \exp 2 \pi i(\mathbf{k} \cdot \mathbf{r})$ (in general a noncentral potential). $\mathbf{R}$ is the field point, $\mathbf{k}$ an arbitrary vector in reciprocal space. Such sums are

and

$$
S(\mathbf{R} \mid \mathbf{k})=\sum_{\lambda_{1}, \lambda_{2}, \lambda_{3}=-\infty}^{+\infty} \phi\left(\mathbf{r}_{\lambda}-\mathbf{R}\right) e^{2 \pi i \mathbf{k} \cdot \mathbf{r} \lambda}
$$

$$
S^{\prime}(\mathbf{R} \mid \mathbf{k})=\Sigma_{\lambda_{1}, \lambda_{2}, \lambda_{s}=-\infty}^{+\infty} \phi\left(\mathbf{r}_{\lambda}-\mathbf{R}\right) e^{2 \pi i \mathbf{k} \cdot \mathbf{r} \lambda} .
$$

$\Sigma$ means that the term $\lambda_{1}=\lambda_{2}=\lambda_{3}=0$ is excluded from the summation. $\sum_{\lambda_{1}, \lambda_{2}, \lambda_{3}}$ will be abbreviated as $\Sigma_{\lambda}$. One has obviously

$$
S(\mathbf{R} \mid \mathbf{k})=S^{\prime}(\mathbf{R} \mid \mathbf{k})+\phi(-\mathbf{R}) .
$$

Since

$$
S\left(\mathbf{R}+\mathbf{r}_{\lambda^{\prime}} \mid \mathbf{k}\right)=\exp \left(2 \pi i \mathbf{k} \cdot \mathbf{r}_{\lambda^{\prime}}\right) S(\mathbf{R} \mid \mathbf{k})
$$

only vectors $\mathbf{R}$ lying within the zero cell need to be considered. Therefore, as we consider simple lattices only, $\mathbf{R}$ will not be equal to any lattice vector except perhaps $\mathbf{R}=0$ (In the latter case $S$ usually has no meaning for the common choices of $\phi$ ). $S$ gives the potential field in a lattice where the origin is occupied, $S^{\prime}$ gives the field in the neighbourhood of an empty site. For $\mathbf{R}=0 S^{\prime}$ is called the self potential of the lattice.

§ 3. The Madelung constant $\alpha_{M}$. In this section we will illustrate the method by applying it to a very simple and well-known case, namely the calculation of the Madelung constant. This constant is defined as the self potential in a lattice where the lattice sites are occupied by alternately positive and negative unit charges

$$
\alpha_{M}=\Sigma_{\lambda}^{\prime} \frac{(-1)^{\lambda_{1}+\lambda_{2}+\lambda_{3}}}{r_{\lambda}} .
$$

The transformation of $\alpha_{M}$ into a more rapidly converging expression $((10)$ is only conditionally convergent) takes place in four steps.

1. (10) is formally written in terms of an integral

$$
\alpha_{M}=\int \frac{w(\mathbf{r})}{r} \mathrm{~d} \mathbf{r}
$$

where

$$
\begin{gathered}
w(\mathbf{r})=\Sigma_{\lambda}^{\prime}(-1)^{\lambda_{1}+\lambda_{2}+\lambda_{\mathbf{3}}} \delta\left(\mathbf{r}-\mathbf{r}_{\lambda}\right)=\Sigma_{\lambda}^{\prime} \exp \left(2 \pi i \mathbf{k}_{\frac{1}{1}} \cdot \mathbf{r}\right) \delta\left(\mathbf{r}-\mathbf{r}_{\lambda}\right) ; \\
\mathbf{k}_{\frac{1}{2}}=\frac{1}{2}\left(\mathbf{b}_{1}+\mathbf{b}_{2}+\mathbf{b}_{\mathbf{3}}\right) \quad(\mathrm{cf} . \text { end of } \S 6) .
\end{gathered}
$$

$\Sigma_{\lambda}^{\prime} \delta\left(\mathbf{r}-\mathbf{r}_{\lambda}\right)$ is a function which has $\delta$-singularities at the lattice points (except at the origin) and which is zero elsewhere. The effect of the function $w(\mathbf{r})$ in the integral (11) is to give the sum (10), by virtue of the definition of the $\delta$-function. 
2. (11) is split into two integrals

$$
\alpha_{M}=\int \frac{w(\mathbf{r})}{r} \Phi(\sqrt{ } \pi r) \mathrm{d} \mathbf{r}+\int \frac{w(\mathbf{r})}{r}\{1-\Phi(\sqrt{ } \pi r)\} \mathrm{d} \mathbf{r} .
$$

$\Phi(x)$ is the complement of the errorfunction

$$
\Phi(x)=(2 / \sqrt{ } \pi) \int_{x}^{\infty} e^{-t^{2}} \mathrm{~d} t=\operatorname{Erfc}(x) .
$$

3. The next step consists in applying Parseval's formula $\left.{ }^{8}\right)$ to the second integral of (13). This formula states that if $F(\mathbf{h})$ and $G(\mathbf{h})$ are the threedimensional Fourier transforms $\left(F T_{3}\right)$ of $f(\mathbf{r})$ and $g(\mathbf{r})$ respectively, then

$$
\int F(\mathbf{h}) G^{*}(\mathbf{h}) \mathrm{d} \mathbf{h}=\int f(\mathbf{r}) g^{*}(\mathbf{r}) \mathrm{d} \mathbf{r} .
$$

Using now

$F T_{3}\{w(\mathbf{r})\}=\left(1 / v_{a}\right) \Sigma_{\lambda} \delta\left\{\mathbf{h}-\left(\mathbf{h}_{\lambda}-\mathbf{k}_{\mathfrak{l}}\right)\right\}-1 \quad$ (cf. appendix 3, (A. 12))

and

$$
F T_{3}\left\{\frac{1-\Phi(\sqrt{\pi} r)}{r}\right\}=\frac{e^{-\pi h^{2}}}{\pi h^{2}} \quad(\text { cf. appendix 4, (A. 19)) }
$$

we find with (15) for the second integral of (13)

$$
\int \frac{w(\mathbf{r})}{r}\{1-\Phi(\sqrt{\pi} r)\} \mathrm{d} \mathbf{r}=\frac{1}{v_{a}} \int\left[\Sigma_{\lambda} \delta\left\{\mathbf{h}-\left(\mathbf{h}_{\lambda}-\mathbf{k}_{\frac{1}{2}}\right)\right\}-1\right] \frac{e^{-\pi h^{2}}}{\pi h^{2}} \mathrm{~d} \mathbf{h} .
$$

4. Finally, the integrals in (13) are "evaluated", leading back to summations. One obtains

$$
\alpha_{M}=\Sigma_{\lambda}^{\prime}(-1)^{\lambda_{1}+\lambda_{2}+\lambda_{3}} \frac{\Phi\left(\sqrt{\pi} r_{\lambda}\right)}{r_{\lambda}}+\frac{1}{\pi v_{a}} \Sigma_{\lambda} \frac{e^{-\pi\left|\mathbf{h}_{\lambda}-\mathbf{k}_{\underline{1}}\right|^{2}}}{\left|\mathbf{h}_{\lambda}-\mathbf{k}_{\underline{\underline{1}}}\right|^{2}}-2
$$

where we used $\int\left\{\exp \left(-\pi h^{2}\right) / \pi h^{2}\right\} \mathrm{d} \mathbf{h}=2$.

This expression is a very convenient one for the evaluation of $\alpha_{M}$; the functions appearing in it have been tabulated extensively ${ }^{9}$ ). Only very few terms of each of both series are needed to give a very accurate value. This expression for $\alpha_{M}$ is equivalent to the one that has been derived by Ewald and Shockley*) for a cubic lattice.

The expression (19) is a rapidly converging one as contrasted to (10) which exhibits a slow conditional convergence only. Yet, going from (10) to (19), no explicit use has been made of any physical argument, leading to a uniquely determined value of $\alpha_{M}$ with the proper physical meaning. It can be shown, however, that the present method is equivalent to a procedure in which the potential field due to all lattice points within a sphere around

\footnotetext{
*) See Kittel ${ }^{10}$ ). If one chooses $\eta=\pi$ in the Ewald-Shockley formula it becomes identical with (19) for the case $v_{a}=1$. (The first summation in the $E-S$ expression, as quoted by Kittel, should be taken over odd values of $k$ only).
} 
the origin is calculated, and where afterwards the limit for infinite jadius of the sphere is taken. This means that the terms are added in order of increasing distance of the lattice points from the origin, which from a physical point of view seems in many cases to be a sound procedure.

$\S 4$. Principle of the summation method. Before applying the summation method to more general types of lattice sums, we will first, by a qualitative reasoning, illustrate the underlying idea of it for the case of a one dimensional series.

Suppose that we have a smooth function $f(x)$ which approaches zero slowly for $x \rightarrow \infty$; $f(0)$ may be either finite or infinite. Then the sum

$$
S=\Sigma_{1}^{\infty} f(n)
$$

if it does exists, will converge slowly.

The proposed method consists in introducing an auxiliary function $\mathfrak{F}(x)$ for which $\mathfrak{F}(0)$ is finite and which approaches zero quickly as $x \rightarrow \infty$ (a third condition follows below). We may write

$$
S=\Sigma_{1}^{\infty} f(n) \mathfrak{F}(n)+\Sigma_{1}^{\infty} f(n)\{1-\mathfrak{F}(n)\} .
$$

The first series in (20) will have good convergence whereas the second series will have the same rate of convergence as the original expression (20). Since the Fourier transform of a smooth function is a function which approaches zero rapidly for increasing argument, we may expect that the "flatter" the function $f(x)\{1-\mathfrak{F}(x)\}$ is, the better the convergence of the Fourier transform of $\sum f(n)\{1-\mathfrak{F}(n)\}$ will be. (Other examples of this property of the Fourier transform are the uncertainty relation in quantum mechanics and the fact that $F T\{\delta(x)\}=1$ and vice versa). The third condition to be imposed on $\mathfrak{F}(x)$, therefore, is that $f(x)\{1-\mathfrak{F}(x)\}$ should be a smooth function in the neighbourhood of $x=0$ (the smoothness further away from the origin being assured by that of $f(x)$ ).

It is clear that the conditions to be fulfilled by $\mathfrak{F}$ by no means determine its choice uniquely but leave room for the choice of a "convenient" form for it, i.e. such a form that conveniently summable series are obtained. This can for instance require that the relevant functions or parts thereof are available in tabulated form.

The above method may be used for the evaluation of one-, two- as well as three-dimensional series (cf. appendix 4).

$\S 5$. Treatment of a more general type of lattice sum. In this section we will treat a more general type of lattice sum, of which the Madelung constant is a special case. The derivation is carried out along exactly the same lines as in $\S 3$, but the single steps in it will be considered in a little more detail. 
The shortness of the particular method of this paper is due to the use of $\delta$-functions in the intermediate steps of the derivation. These $\delta$-functions will be employed in the way that is customary in physics, without any claim of mathematical rigour.

The lattice sum to be considered in this section is

$$
S^{\prime}(\mathbf{R} \mid \mathbf{k}, n)=\Sigma_{\lambda}^{\prime} \frac{e^{2 \pi i \mathbf{k} \cdot \mathbf{r} \lambda}}{\left|\mathbf{r}_{\lambda}-\mathbf{R}\right|^{2 n}}
$$

In principle $n$ can be any real number for which (22) exists (cf., however, the end of this section). Examples of lattice sums of this type have been treated extensively in the literature (e.g. the cases $\mathbf{k}=0$ and $\mathbf{k}_{\frac{1}{2}}$ ). It will be considered here to illustrate the general method which has been outlined in the preceding section.

The first step again consists in expressing $S^{\prime}$ formally in an integral

$$
S^{\prime}(\mathbf{R} \mid \mathbf{k}, n)=\int \frac{w(\mathbf{r}, \mathbf{k})}{|\mathbf{r}-\mathbf{R}|^{2 n}} \mathrm{~d} \mathbf{r}
$$

with

$$
w(\mathbf{r}, \mathbf{k})=\Sigma_{\lambda}^{\prime} \delta\left(\mathbf{r}-\mathbf{r}_{\lambda}\right) \exp (2 \pi i \mathbf{k} \cdot \mathbf{r}) .
$$

The next step is to introduce a suitable auxiliary function $\mathfrak{F}_{1}$ and to split (23) into two parts according to (21). A convenient choice is

$$
\widetilde{F}_{1}(\mathbf{r}-\mathbf{R})=\Gamma\left(n, \pi|\mathbf{r}-\mathbf{R}|^{2}\right) / \Gamma(n) .
$$

Then

$$
1-\mathfrak{F}_{1}(\mathbf{r}-\mathbf{R})=\gamma\left(n, \pi|\mathbf{r}-\mathbf{R}|^{2}\right) / \Gamma(n) .
$$

$\Gamma(n, x)$ is the incomplete gammafunction $\left.{ }^{11}\right)$ defined by

$$
\begin{gathered}
\Gamma(n, x)=\int_{x}^{\infty} e^{-t} t^{n-1} \mathrm{~d} t \\
\gamma(n, x)=\Gamma(n)-\Gamma(n, x)=\int_{0}^{x} e^{-t} t^{n-1} \mathrm{~d} t .
\end{gathered}
$$

From (25), (26) and (27) it is easily verified that this $\mathfrak{F}_{1}$ satisfies the three conditions mentioned in $\S 4$.

We can now write (23)

$S^{\prime}(\mathbf{R} \mid \mathbf{k}, n)=$

$=\frac{1}{\Gamma(n)}\left[\int w(\mathbf{r}, \mathbf{k}) \frac{\Gamma\left(n, \pi|\mathbf{r}-\mathbf{R}|^{2}\right)}{|\mathbf{r}-\mathbf{R}|^{2 n}} \mathrm{~d} \mathbf{r}+\int w(\mathbf{r}, \mathbf{k}) \frac{\gamma\left(n, \pi|\mathbf{r}-\mathbf{R}|^{2}\right)}{|\mathbf{r}-\mathbf{R}|^{2 n}} \mathrm{~d} \mathbf{r}\right]$.

It will be convenient to write in the second integral

hence

$$
w(\mathbf{r}, \mathbf{k})=\bar{w}(\mathbf{r}, \mathbf{k})-\delta(\mathbf{r}) \exp (2 \pi i \mathbf{k} \cdot \mathbf{r})
$$

$$
\bar{w}(\mathbf{r}, \mathbf{k})=\Sigma_{\lambda} \delta\left(\mathbf{r}-\mathbf{r}_{\lambda}\right) \exp (2 \pi i \mathbf{k} \cdot \mathbf{r})
$$


At this stage we apply Parseval's formula to the second integral.of (29). Using

$$
F T_{\mathbf{3}}[\bar{w}(\mathbf{r}, \mathbf{k})]=\left(1 / v_{a}\right)\{\sigma(\mathbf{h}+\mathbf{k})+\delta(\mathbf{h}+\mathbf{k})\},
$$

where

$$
\sigma(\mathbf{h}+\mathbf{k})=\Sigma_{\lambda}^{\prime} \delta\left\{\mathbf{h}-\left(\mathbf{h}_{\lambda}-\mathbf{k}\right)\right\}
$$

(cf. appendix (A. 12)), and making use of (30), we can write for (29)

$$
\begin{aligned}
S^{\prime}(\mathbf{R} \mid \mathbf{k}, n) & =\frac{1}{\Gamma(n)}\left[\int w(\mathbf{r}, \mathbf{k}) \frac{\Gamma\left(n, \pi|\mathbf{r}-\mathbf{R}|^{2}\right)}{|\mathbf{r}-\mathbf{R}|^{2 n}} \mathrm{~d} \mathbf{r}-\right. \\
& -\int \delta(\mathbf{r}) e^{2 \pi i \mathbf{k} \cdot \mathbf{r}} \frac{\gamma\left(n, \pi|\mathbf{r}-\mathbf{R}|^{2}\right)}{|\mathbf{r}-\mathbf{R}|^{2 n}} \mathrm{~d} \mathbf{r}+ \\
& \left.+\frac{1}{v_{a}} \int\{\sigma(\mathbf{h}+\mathbf{k})+\delta(\mathbf{h}+\mathbf{k})\} F T_{3}\left[\frac{\gamma\left(n, \pi|\mathbf{r}-\mathbf{R}|^{2}\right)}{|\mathbf{r}-\mathbf{R}|^{2 n}}\right] \mathrm{d} \mathbf{r}\right] .
\end{aligned}
$$

From applying (A. 4) to (A. 17) (see appendix) it follows that

$$
F T_{3}\left[\frac{\gamma\left(n, \pi|\mathbf{r}-\mathbf{R}|^{2}\right)}{|\mathbf{r}-\mathbf{R}|^{2 n}}\right]=\pi^{2 n-3 / 2} h^{2 n-3} \Gamma\left(-n+\frac{3}{2}, \pi h^{2}\right) e^{2 \pi i \mathbf{h} \cdot \mathbf{R}} .
$$

Furthermore

$$
\int \delta(\mathbf{r}) e^{2 \pi i \mathbf{k} \cdot \mathbf{r}} \frac{\gamma\left(n, \pi|\mathbf{r}-\mathbf{R}|^{2}\right)}{|\mathbf{r}-\mathbf{R}|^{2 n}} \mathrm{~d} \mathbf{r}=\left\{\begin{array}{l}
\gamma\left(n, \pi R^{2}\right) / R^{2 n} \text { for } \mathbf{R} \neq 0 \\
\pi^{n / n} \text { for } \mathbf{R}=0, n>0
\end{array}\right.
$$

and

$$
\begin{aligned}
& \int \delta(\mathbf{h}+\mathbf{k}) h^{2 n-3} \Gamma\left(-n+\frac{3}{2}, \pi h^{2}\right) e^{2 \pi i \mathbf{h} \cdot \mathbf{R}} \mathrm{d} \mathbf{h}= \\
& \quad=\left\{\begin{array}{l}
k^{2 n-3} \Gamma\left(-n+\frac{3}{2}, \pi k^{2}\right) \exp (-2 \pi i \mathbf{k} \cdot \mathbf{R}) \text { for } \mathbf{k} \neq 0 \\
\pi^{-n+3 / 2} /\left(n-\frac{3}{2}\right) \quad \text { for } \mathbf{k}=0, n>\frac{3}{2} .
\end{array}\right.
\end{aligned}
$$

Making use of these relations and carrying out step four, i.e. replacing the integrals by the summations which they represent, we finally find as the general expression for (22)

$$
\begin{aligned}
& S^{\prime}(\mathbf{R} \mid \mathbf{k}, n)=\frac{1}{\Gamma(n)}\left[\Sigma_{\lambda}^{\prime} \frac{\Gamma\left(n, \pi\left|\mathbf{r}_{\lambda}-\mathbf{R}\right|^{2}\right)}{\left|\mathbf{r}_{\lambda}-\mathbf{R}\right|^{2 n}} e^{2 \pi i \mathbf{k} \cdot \mathbf{r} \lambda}-\frac{\gamma\left(n, \pi R^{2}\right)}{R^{2 n}}+\right. \\
& \left.\quad+\frac{\pi^{2 n-3 / 2}}{v_{a}} \Sigma_{\lambda}\left|\mathbf{h}_{\lambda}-\mathbf{k}\right|^{2 n-3} \Gamma\left(-n+\frac{3}{2}, \pi\left|\mathbf{h}_{\lambda}-\mathbf{k}\right|^{2}\right) e^{2 \pi i\left(\mathbf{h} \lambda^{-\mathbf{k}}\right) \cdot \mathbf{R}}\right] .
\end{aligned}
$$

The expression in this form holds for $\mathbf{R} \neq 0$ and $\mathbf{k} \neq 0$. For the case $\mathbf{R}=0$ $(\mathbf{k} \neq 0)$ the second term in the right hand side should be replaced by its limit value $\pi^{n} / n$; the series then only converges for $n>0$. For $\mathbf{k}=0$ the second summation should carry a prime and the term $\pi^{n} / v_{a}\left(n-\frac{3}{2}\right)$ should be added. In that case the sum only exists for $n>\frac{3}{2}$. 
Instead of $\pi$ as a factor in the argument of the incomplete gamma function (cf. (25)), any arbitrary number $\alpha$ could of course have been choosen. However, the particular choice $\alpha=\pi$ leads, as can be verified from the asymptotic expansion for the incomplete gamma function, to the same rapidity of convergence for both series in (38).

The incomplete gamma function $\Gamma(n, x)$ has been tabulated for various values of $n$ (cf. ref. 12 p. 206 and ref. 13). In the most commonly occurring case $n$ is either integer or half integer. Because of the recurrence relation

$$
\Gamma(n+1, x)=n \Gamma(n, x)+x^{n} e^{-x},
$$

$\Gamma(n, x)$ then can always be related to

$$
\Gamma(1, x)=e^{-x} \text { or } \Gamma(0, x)=-E i(-x)
$$

and

$$
\Gamma\left(\frac{1}{2}, x\right)=\sqrt{\pi} \operatorname{Erfc}(\sqrt{x})
$$

respectively. These functions have been tabulated extensively ${ }^{9}{ }^{12}$ ).

\$6. Treatment of the lattice sum

$$
S_{l m}^{\prime}(o \mid \mathbf{k}, n)=\Sigma_{\lambda}^{\prime} \frac{Y_{l m}(0, \phi) e^{2 \pi i \mathbf{k} \cdot \mathbf{r} \lambda}}{r_{\lambda}^{2 n}+l} \quad\left\{\begin{array}{c}
l=0,1,2, \ldots \\
m=0, \pm 1, \ldots, \pm l
\end{array}\right\},
$$

where we employed the usual notation for spherical harmonics:

$Y_{l m}(\theta, \phi)=(2 \pi)^{-\frac{1}{2}} \overline{P_{l}^{m}}(\cos \theta) e^{i m \phi} . \overline{P_{l}^{m}}$ is the normalized associated Legendre function. $\theta$ and $\phi$ are the polar angles of $\mathbf{r}_{\lambda}$ with respect to an arbitrarily chosen cartesian coordinate system.

The sum (39) is the self potential corresponding to $S_{l m}^{\prime}(\mathbf{R} \mid \mathbf{k}, n)$ of which $S^{\prime}(\mathbf{R} \mid \mathbf{k}, n)(22)$ is the special case $l=0$. For simplicity we will restrict our considerations here to sums of the type (39). The extension to the case $\mathbf{R} \neq 0$ is straightforward.

We will not carry out the derivation, which is completely analogous to that of $\S 5$, in detail but only write down the most important formulae. A convenient form for the auxiliary function $\mathfrak{F}_{2}$ is

$$
\mathfrak{F}_{2}(\mathbf{r})=\Gamma\left(n+l, \pi r^{2}\right) / \Gamma(n+l) .
$$

It is shown in the appendix (A. 13) that

$$
\begin{aligned}
& F T_{3}\left[\frac{\gamma\left(n+l, \pi r_{2}\right) Y_{l m}(\theta, \phi)}{r^{2 n+l}}\right]= \\
& \quad=i^{l} \pi^{2 n+l-3 / 2} h^{2 n+l-3} \Gamma\left(-n+\frac{3}{2}, \pi h^{2}\right) Y_{l m}(\vartheta, \varphi),
\end{aligned}
$$

where $\vartheta$ and $\varphi$ are the polar angles of the vector $\mathbf{h}$, taken with respect to the 
same coordinate system as $\theta$ and $\phi$. Furthermore it is easily verified that

$$
\int \delta(\mathbf{r}) e^{2 \pi i \mathbf{k} \cdot \mathbf{r}} \frac{\gamma\left(n+l, \pi r^{2}\right)}{r^{2 n+l}} Y_{l m}(\theta, \phi) \mathrm{d} \mathbf{r}=0 \text { for } l>0, n+l>0
$$

and that

$$
\begin{aligned}
& \int \delta(\mathbf{h}+\mathbf{k}) h^{2 n+l-3} \Gamma\left(-n+\frac{3}{2}, \pi h^{2}\right) Y_{l m}(\vartheta, \varphi) \mathrm{d} \mathbf{h}= \\
& \quad=\left\{\begin{array}{l}
k^{2 n+l-3} \Gamma\left(-n+\frac{3}{2}, \pi k^{2}\right)(-1)^{l} Y_{l m}\left(\vartheta_{\mathbf{k}}, \varphi_{\mathbf{k}}\right) \text { for } \mathbf{k} \neq 0 \\
0 \text { for } \mathbf{k}=0, l>0, n>\frac{3}{2}
\end{array}\right.
\end{aligned}
$$

$\vartheta_{\mathbf{k}}$ and $\varphi_{\mathbf{k}}$ are the polar angles of $\mathbf{k}$.

Using (41), (42) and (43a) we have for the case $\mathbf{k} \neq 0$

$$
\begin{aligned}
& S_{l, m}^{\prime}(o \mid \mathbf{k}, n)=\frac{1}{\Gamma(n+l)}\left[\Sigma_{\lambda}^{\prime} \frac{\Gamma\left(n+l, \pi r_{\lambda}^{2}\right) Y_{l m}(\theta, \phi) e^{2 \pi i \mathbf{k} \cdot \mathbf{r} \lambda}}{r_{\lambda}^{2 n+l}}+\right. \\
& \left.+\frac{i^{l} \pi^{2 n+l-3 / 2}}{v_{a}} \Sigma_{\lambda}\left|\mathbf{h}_{\lambda}-\mathbf{k}\right|^{2 n+l-3} \Gamma\left(-n+\frac{3}{2}, \pi\left|\mathbf{h}_{\lambda}-\mathbf{k}\right|^{2}\right) Y_{l m}\left(\vartheta_{\mathbf{h} \lambda-\mathbf{k}}, \varphi_{\mathbf{h} \lambda-\mathbf{k}}\right)\right] . \\
& (l>0, n+l>0)
\end{aligned}
$$

For $\mathbf{k}=0$ the second summation should be read with a prime; the sum then only exists for $n>\frac{3}{2}$ (cf. (43b)). This is also the case when $\mathbf{k}$ is equal to any other reciprocal lattice vector, as is easily verified.

Knowledge of lattice sums of the type (39) is of interest for various problems. If, for instance, one expands the potential field around an arbitrary lattice point of an ionic lattice in terms of spherical harmonics, the coefficients of this expansion can be expressed in terms of the sums $S_{l m}^{\prime}\left(o \mid \mathbf{k}_{1}, \frac{1}{2}\right)$, as can be shown very easily. Of course this potential is also simply $S^{\prime}\left(\mathbf{R} \mid \mathbf{k}_{\frac{1}{2}}, \frac{1}{2}\right)$, but as this sum involves the distances $\mathbf{r}_{\lambda}-\mathbf{R}$, it is sometimes less convenient. This is just a simple example of a general connection, existing between the lattice sums $S^{\prime}(\mathbf{R} \mid \mathbf{k}, n), S_{l n}^{\prime}(\mathbf{R} \mid \mathbf{k}, n)$ and $S_{l m}^{\prime}(o \mid \mathbf{k}, n)$, to which we hope to come back in a subsequent paper.

In some cases the sums (39) can, without, any calculation, be shown to vanish on account of the symmetry of the lattice considered. This holds in particular for the sums $S_{l m}^{\prime}(0 . \mid 0, n)$. Since, for example, in a Bravais lattice the origin is a center of inversion and since further $Y_{l m}\left(\theta^{\prime}, \phi^{\prime}\right)=$ $=(-1)^{l} Y_{l m}(\theta, \phi)\left(\theta^{\prime}=\pi-\theta\right.$ and $\phi^{\prime}=\pi+\phi$ being the inversion angles of $\theta$ and $\phi$ ) all $S_{l m}^{\prime}(o \mid o, n)$ will vanish for odd $l$, because of a complete cancellation of terms. This is true independent of any additional symmetry of the lattice. An other example is that of the cubic lattice, where the question which sums $S_{l m}^{\prime}(0 \mid 0, n)$ vanish and which do not, is obviously closely related to the well-known problem of the cubic spherical harmonics, i.e. the problem which linear combinations of $Y_{l m}$ 's have the cubic symmetry. 
Remark on the use of the vector $\mathbf{k}$ (cf. (7), (8)). The freedom in the choice of the reciprocal space vector $\mathbf{k}$, appearing in the factor $\exp \left(2 \pi i \mathbf{k} \cdot \mathbf{r}_{\lambda}\right)$ in the general lattice sums (22) and (39), allows for the description of a wide variety of situations by these two expressions. that

Using $\mathbf{r}_{\lambda}=\sum_{i} \lambda_{i} \mathbf{a}_{i}$ and $\mathbf{k}=\sum_{i} k_{i} \mathbf{b}_{i}(i=1,2,3)$ it follows from $\mathbf{a}_{i} \cdot \mathbf{b}_{i}=\delta_{i j}$

$$
\mathbf{k} \cdot \mathbf{r}_{\lambda}=\sum_{i} k_{i} \lambda_{i} .
$$

The special choice $\mathbf{k}=\frac{1}{2}\left(\mathbf{b}_{1}+\mathbf{b}_{2}+\mathbf{b}_{3}\right)$ leads, as follows immediately from (45) to $\exp \left(2 \pi i \mathbf{k} \cdot \mathbf{r}_{\lambda}\right)=(-1)^{\lambda_{1}+\lambda_{2}+\lambda_{3}}$, which can be applied to the case of an ionic lattice (cf. $\S 3$ ). More generally $\mathbf{k}$ can be chosen in such a way as to describe lattices in which the charges or multipoles vary in a periodic way as regards magnitude or orientation.

\section{APPENDICES}

1. Lattice sums and Fourier transform. The three-dimensional Fourier transform of a function $f(\mathbf{r})$ is defined as

$$
F T_{\mathbf{3}}\{f(\mathbf{r})\}=\int f(\mathbf{r}) \exp (2 \pi i \mathbf{h} \cdot \mathbf{r}) \mathrm{d} \mathbf{r} \equiv F(\mathbf{h}),
$$

the inversion is

$$
F T_{3}\{F(\mathbf{h})\}=\int F(\mathbf{h}) \exp (-2 \pi i \mathbf{h} \cdot \mathbf{r}) \mathrm{d} \mathbf{h}=f(\mathbf{r}) .
$$

From (A. 1) it follows in particular that

and

$$
F T_{3}\{f(\mathbf{r}) \exp (2 \pi i \mathbf{k} \cdot \mathbf{r})\}=F(\mathbf{h}+\mathbf{k})
$$

$$
F T_{\mathbf{3}}\{t(\mathbf{r}-\mathbf{R})\}=F(\mathbf{h}) \exp (2 \pi i \mathbf{h} \cdot \mathbf{R}) .
$$

A well-known formal expression for the $\delta$-function is

$$
\delta(\mathbf{r}-\boldsymbol{\xi})=\int \exp \{-2 \pi i \mathbf{h} \cdot(\mathbf{r}-\boldsymbol{\xi})\} \mathrm{d} \mathbf{h},
$$

for which we clearly can write

By inversion

$$
\delta(\mathbf{r}-\boldsymbol{\xi})=F T_{3}\{\exp (2 \pi i \mathbf{h} \cdot \boldsymbol{\xi})\} .
$$

$$
F T_{\mathbf{3}}\{\delta(\mathbf{r}-\boldsymbol{\xi})\}=\exp (2 \pi i \mathbf{h} \cdot \boldsymbol{\xi}) .
$$

In a similar fashion one can derive the Fourier transform of the function $\Sigma_{\lambda} \delta\left(\mathbf{r}-\mathbf{r}_{\lambda}\right)$, which has $\delta$-singularities at the lattice points and which is zero elsewhere. One finds

$$
F T_{3}\left\{\Sigma_{\lambda} \delta\left(\mathbf{r}-\mathbf{r}_{\lambda}\right)\right\}=\Sigma_{\lambda} \exp \left(2 \pi i \mathbf{h} \cdot \mathbf{r}_{\lambda}\right) .
$$

The sum in the right hand side of (A.7) is, except for the multiplying factor $1 / v_{a}$, presicely a summation of $\delta$-functions over the reciprocal lattice points. 
2. Proof of the relation

$$
\Sigma_{\lambda} \exp \left(2 \pi i \mathbf{h} \cdot \mathbf{r}_{\lambda}\right)=\left(1 / v_{a}\right) \Sigma_{\lambda} \delta\left(\mathbf{h}-\mathbf{h}_{\lambda}\right) .
$$

We start out from Poisson's sum formula ${ }^{14}$ )

$$
\Sigma_{n=-\infty}^{+\infty} f(x-n)=\sum_{\nu=-\infty}^{+\infty} \exp (2 \pi i v x) \int_{-\infty}^{+\infty} f(\xi) \exp (-2 \pi i \nu \xi) \mathrm{d} \xi \text {. (A. 9) }
$$

Inserting for $f(x)$ the function $\delta(x)$ we find

$$
\Sigma_{n=-\infty}^{+\infty} \delta(x-n)=\Sigma_{\nu=-\infty}^{+\infty} \exp (2 \pi i v x) .
$$

Using $\mathbf{r}_{\lambda}=\sum_{i} \lambda_{i} \mathbf{a}_{i} \quad(i=1,2,3)$ we can write for the left hand side of (A. 8)

$$
\Sigma_{\lambda} \exp \left(2 \pi i \mathbf{h} \cdot \mathbf{r}_{\lambda}\right)=\Sigma_{\lambda} \exp \left\{2 \pi i \Sigma_{i} \lambda_{i}\left(\mathbf{h} \cdot \mathbf{a}_{i}\right)\right\}
$$

which, by virtue of (A. 10), leads to

$$
\Sigma_{\lambda} \exp \left(2 \pi i \mathbf{h} \cdot \mathbf{r}_{\lambda}\right)=\Sigma_{\lambda}\left\{\Pi_{i} \delta\left(\mathbf{h} \cdot \mathbf{a}_{i}-\lambda_{i}\right)\right\} .
$$

The integers $\lambda_{i}(i=1,2,3)$ may be written as $\lambda_{i}=\mathbf{h}_{\lambda} \cdot \mathbf{a}_{i}$, as follows immediately from (4) and (6). We thus can write

$$
\Sigma_{\lambda} \exp \left(2 \pi i \mathbf{h} \cdot \mathbf{r}_{\lambda}\right)=\Sigma_{\lambda}\left(\Pi_{i} \delta\left\{\left(\mathbf{h}-\mathbf{h}_{\lambda}\right) \cdot \mathbf{a}_{i}\right\}\right] .
$$

Let the basisvector $\mathbf{a}_{i}$ have the cartesian components $a_{i j}$, then the 9 numbers $a_{i j}(i, j=1.2,3)$ determine a transformation, the matrix of which we will denote by $\mathbf{A}$. Then the numbers $\mathbf{h} \cdot \mathbf{a}_{i}(i=1,2,3)$ are the components of the vector $\mathbf{A} \mathbf{h}$ which results from applying the transformation $\mathbf{A}$ to $\mathbf{h}$. Using $\delta(\mathbf{r})=\delta(x) \delta(y) \delta(z)$ we can writte, therefore,

$$
\Sigma_{\lambda} \exp \left(2 \pi i \mathbf{h} \cdot \mathbf{r}_{\lambda}\right)=\Sigma_{\lambda}\left[\delta\left\{\mathbf{A}\left(\mathbf{h}-\mathbf{h}_{\lambda}\right)\right\}\right] .
$$

If we now use $\delta(\mathbf{A x})=(1 /|\operatorname{det} \mathbf{A}|) \delta(\mathbf{x})$ and $|\operatorname{det} \mathbf{A}|=v_{a}$ we arrive at

$$
\Sigma_{\lambda} \exp \left(2 \pi i \mathbf{h} \cdot \mathbf{r}_{\lambda}\right)=\left(1 / v_{a}\right) \Sigma_{\lambda} \delta\left(\mathbf{h}-\mathbf{h}_{\lambda}\right) .
$$

This completes the proof of (A. 8). Finally, from (A. 7) and (A. 8)

$$
F T_{\mathbf{3}}\left\{\Sigma_{\lambda} \delta\left(\mathbf{r}-\mathbf{r}_{\lambda}\right)\right\}=\left(1 / v_{a}\right) \Sigma_{\lambda} \delta\left(\mathbf{h}-\mathbf{h}_{\lambda}\right) .
$$

Applying (A. 3) to (A. 11) one finds

$$
F T_{\mathbf{3}}\left[\Sigma_{\lambda} \delta\left(\mathbf{r}-\mathbf{r}_{\lambda}\right) \exp (2 \pi i \mathbf{k} \cdot \mathbf{r})\right]=\left(1 / v_{a}\right) \Sigma_{\lambda} \delta\left(\mathbf{h}+\mathbf{k}-\mathbf{h}_{\lambda}\right) .
$$

\section{Proof of the relation}

$$
\begin{aligned}
& F T_{3}\left[\frac{\gamma\left(n+l, \pi r^{2}\right) Y_{l m}\left(\theta_{\mathbf{r}}, \phi_{\mathbf{r}}\right)}{r^{2 n+l}}\right]= \\
& =i l^{2 n+l--^{3} / 2} h^{2 n+l-3} \Gamma\left(-n+\frac{3}{2}, \pi h^{2}\right) Y_{l m}\left(\vartheta_{\mathbf{k}}, \varphi_{\mathbf{b}}\right) .
\end{aligned}
$$

We will here give the proof of the reverse statement, viz. that the $F T_{3}$ of the right hand side is equal to the bracket expression on the left hand side. 
One can easily show that ${ }^{15}$ )

$F T_{3}\left[g(h) Y_{l m}\left(\vartheta_{\mathbf{h}}, \varphi_{\mathbf{h}}\right)\right]=2 \pi(-i)^{l} Y_{l m}\left(\theta_{\mathbf{r}}, \phi_{\mathbf{r}}\right) r^{-1 / 2} \int_{0}^{\infty} g(h) J_{l+1 / 2}(2 \pi h r) h^{3 / 2} \mathrm{~d} h$.

Applying this to the left hand side of (A. 13) we have $F T_{3}\left[i \pi^{2 n+l-^{3} / 2} h^{2 n+l-3} \Gamma\left(-n+\frac{3}{2}, \pi h^{2}\right) Y_{l m}\left(\vartheta_{\mathbf{h}}, \varphi_{\mathbf{h}}\right)\right]=$

$$
=B r^{-1 / 2} \int_{0}^{\infty} h^{2 n+l-3 / 2} \Gamma\left(-n+\frac{3}{2}, \pi h^{2}\right) J_{l+1 / 2}(2 \pi h r) \mathrm{d} h \equiv I_{1}
$$

where

$$
B=2 \pi^{2 n+l-1 / 2} Y_{l m}\left(\theta_{\mathbf{r}}, \phi_{\mathbf{r}}\right) .
$$

Inserting the expression for the incomplete gammafunction (cf. (27)) we have

$$
\begin{aligned}
I_{1} & =B r^{-1 / 2} \int_{0}^{\infty} \mathrm{d} h h^{2 n+l^{-3 / 2}} J_{l+1 / 9}(2 \pi h r) \int_{\pi h^{2}}^{\infty} e^{-x} x^{-n} \mathrm{~d} x= \\
& =\frac{B}{(2 \pi)^{2 n+l}--^{1 / 2} r^{2 n+l}} \int_{0}^{\infty} e^{-x} x^{-n+^{1 / 2}} \mathrm{~d} x \int_{0}^{2 r^{\prime} \pi x} t^{2 n+l-s / 2} J_{l+1 / 2}(t) \mathrm{d} t . \text { (A. 14) }
\end{aligned}
$$

This must be shown to be equal to

$$
I_{2}=\frac{\gamma\left(n+l, \pi r^{2}\right) Y_{l m}\left(\theta_{\mathrm{r}}, \phi_{\mathrm{r}}\right)}{r^{2 n+l}}=\frac{Y_{l m}\left(\theta_{\mathrm{r}}, \phi_{\mathrm{r}}\right)}{r^{2 n+l}} \int_{0}^{\pi r^{2}} e^{-x} x^{n+l-1} \mathrm{~d} x .
$$

In other words we must prove the equality

$$
r^{2 n+l} I_{1}=r^{2 n+l} I_{2} .
$$

It is obvious that for $r=0$ the equality holds. If we now can prove that the derivatives of both sides of (A. 16) with respect to $r$ are equal then relation (A. 13) is established.

One finds after a short derivation

$$
\begin{array}{r}
\frac{d}{\mathrm{~d} r}\left(r^{2 n+l} I_{1}\right)=B \pi^{-n-1 / 3 l+1 / \iota} r^{2 n+l-3 / 2} \int_{0}^{\infty} e^{-u^{2}} u l^{l+3 / 2} J_{l+1 / 2}(2 r \sqrt{\pi} u) \mathrm{d} u= \\
=2 \pi^{n+l} r^{2 n+2 l-1} Y_{l m}\left(\theta_{\mathbf{r}}, \phi_{\mathbf{r}}\right) e^{-\pi r^{2}}, \quad\left(l>-\frac{3}{2}\right) .
\end{array}
$$

For the value of the integral see for instance reference $11 \mathrm{p} .177$. It is easily seen that $(d / \mathrm{d} r)\left(r^{2 n+l} I_{2}\right)$ leads to the same expression. This completes the proof of (A. 13).

Since the proof holds for all $l>-\frac{3}{2}$ we have for $l=0$

$$
F T_{3}\left[\gamma\left(n, \pi r^{2}\right) / r^{2 n}\right]=\pi^{2 n-3 / 2} h^{2 n-3} \Gamma\left(-n+\frac{3}{2}, \pi h^{2}\right) .
$$

4. Note on evaluation of one- and two-dimensional series. The method, employed in this paper for the evaluation of three-dimensional series, may as 
well be used for the evaluation of one- and two- dimensional series. For sums of the type (22) but now one- or two-dimensional, the same auxiliary function as was used in the three-dimensional case, viz. $\mathfrak{F}(r)=\Gamma\left(n, \pi r^{2}\right) / \Gamma(n)$ can be employed. For reference we give the one- and two-dimensional Fourier transforms of the relevant functions

$$
\begin{aligned}
& F T_{1}\left\{\gamma\left(n, \pi r^{2}\right) / r^{2 n}\right\}=\pi^{2 n-1 / 2}|h|^{2 n-1} \Gamma\left(-n+\frac{1}{2}, \pi h^{2}\right) \\
& F T_{2}\left\{\gamma\left(n, \pi r^{2}\right) / r^{2 n}\right\}=\pi^{2 n-1} h^{2 n-2} \Gamma\left(-n+1, \pi h^{2}\right) .
\end{aligned}
$$

The derivation of these relations is completely analogous to that of (A. 17), with which they should be compared.

Received 26-1-57.

\section{REFERENCES}

1) Madelung, E., Pliys. Z. 19 (1918) 524.

2) Ornstein, L. S. and Zernike, F., Proc Kon. Akad. Wetensch. Amsterdam 21 (1918) 911.

3) Ewald, P. P., Ann. Physik 64 (1921) 253.

4) Evjen, H. M., Phys. Rev. 39 (1932) 675.

5) Misra, R. D., Proc. Camb. Phil. Soc. 36 (1940) 173.

6) Placzek, G., Nijboer, B. R. A. and Van Hove, L., Phys. Rev. 82 (1951) 392.

7) Bertaut, F., J. Phys. Radium 13 (1952) 499.

8) Cf. for instance: Titchmarsh, E. C., Introduction to the Theory of Fourier Integrals, 2 nd. ed. Oxford 1948 , p. 50.

9) Cf. Tables of the Error Function and its Derivative, National Bureau of Standards, Applied Mathematics Series 41 .

10) Kittel, C., Introduction to solid State Physics, John. Wiley, New York 1953, p. 347 ff.

11) Cf. for instance, Magnus, W., Oberhettinger, F., Formeln und Sätze etc. Springer, Berlin 1948 , p. 125.

12) Fletcher, A., Miller, J. C. P., Rosenthead, L., An Index of mathematical Tables, McGrawHill, New-York 1946.

13) Pearson, K., Tables of the incomplete Gamma Function, Cambridge 1922 (reissued 1951).

14) Cf. for instance: Madelung, E., Die math. Hilfsmittel des Physikers, Springer, Berlin 1950, p. 69.

15) Cf. Sneddon, I. N., Fourier Transforms, McGraw-Hill, New York 1951, p. 366 ff. 